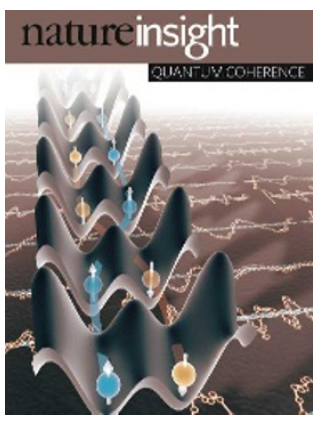

Cover illustration The entangling of atoms through spin coupling in a double-well potential (Courtesy of I. Bloch)

\section{Editor, Nature}

Philip Campbell

Insights Publisher

Steven Inchcoombe

Insights Editor

Karl Ziemelis

Production Editor

Davina Dadley-Moore

Senior Art Editor

Martin Harrison

Art Editor

Nik Spencer

Sponsorship

Amélie Pequignot

Production

Jocelyn Hilton

Marketing

Katy Dunningham

Elena Woodstock

Editorial Assistant

Alison McGill

\title{
QUANTUM COHERENCE
}

Q uantum physics has come a long way since its theoretical beginnings in the early twentieth century. Techniques to manipulate light and matter have become increasingly sophisticated, facilitating fundamental studies of quantum effects and inspiring new technologies. From atomic networks to semiconductor 'spintronics', seemingly disparate areas of research are being driven by a shared goal - to harness and exploit quantum coherence and entanglement.

Inevitably, these laboratory endeavours have necessitated a new theoretical toolbox. The image of a pair of photons zooming off in opposite directions, each sensitive to the other through their quantum entanglement, is conceptually tidy. But what happens when describing the quantum properties of more complex systems? This Insight on quantum coherence and entanglement starts with a Progress article that addresses the problem of 'thinking big': how can entanglement be quantified or measured in a system that comprises many particles and degrees of freedom?

The reviews in this Insight highlight the exciting experimental progress in such systems, covering a wide range of physical settings. They describe both bottom-up approaches, in which researchers strive to achieve increasingly complex systems starting from a very small number of particles and degrees of freedom, and top-down approaches, in which the individual and collective degrees of freedom in larger systems are controlled. Ultimately, the goal is to control many-particle systems at the quantum limit, an attractive prospect for quantum simulation and information applications.

As such, this Insight brings together varied research. We trust, however, that you will find coherence in this diversity.

\section{PROGRESS}

1004

Quantifying entanglement in macroscopic systems

V. Vedral

\section{REVIEWS}

1008 Entangled states of trapped atomic ions

R. Blatt \& D. Wineland

1016 Quantum coherence and entanglement with ultracold atoms in optical lattices

I. Bloch

1023 The quantum internet

H. J. Kimble

1031 Superconducting quantum bits

J. Clarke \& F. K. Wilhelm

1043 Coherent manipulation of single spins in semiconductors

R. Hanson \& D. D. Awschalom

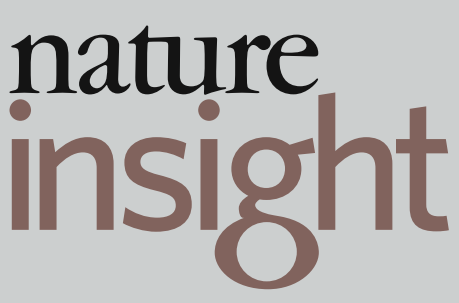

